\title{
The effects of galactic winds on the IGM in semi-analytic simulations
}

\author{
Serena Bertone ${ }^{1,2}$, Simon D. M. White ${ }^{2}$, and Felix Stoehr ${ }^{3}$ \\ ${ }^{1}$ Astronomy Centre, University of Sussex, \\ Falmer, Brighton BN1 9QH, United Kingdom \\ email: s.bertone@sussex.ac.uk \\ ${ }^{2}$ Max Planck Institut für Astrophysik, \\ Karl Schwarzschild Str. 1, 85741 Garching bei München, Germany \\ ${ }^{3}$ Institute d'Astrophysique de Paris, \\ 98bis, bd Arago, 75014 Paris, France
}

\begin{abstract}
We present a new semi-analytic treatment of the evolution of galactic winds within high resolution, large scale cosmological N-body simulations of a $\Lambda$ CDM Universe. We focus our analysis on the impact of winds on the diffuse intergalactic medium at $z=3$ and we make predictions for the volume filling factor of winds as a function of our model parameters. We then verify this prediction by extracting a set of synthetic spectra along random lines of sight through our simulated box and by calculating the probability distribution function (PDF) of the spectral flux. We find that galactic winds do not significantly modify the PDF. We finally argue that the increased flux transmissivity found by Adelberger et al. (2003) around a small sample of Lyman break galaxies may be explained by the presence of hot ionised bubbles due to pressure-driven winds outflowing from the galaxies. However, this effect cannot be explained by cooled, momentum-driven winds. We conclude that the result of Adelberger et al. (2003) may be the outcome of a selection effect.
\end{abstract}

\section{Introduction}

In the last fifteen years, observations of the spectra of high redshift quasars have provided a wealth of data on properties of the intergalactic medium (IGM) which were otherwise impossible to determine. This has dramatically increased our knowledge of the evolution of the Universe and has demonstrated that present models of structure and galaxy formation need to include more physics to successfully reproduce the observational results and to be able to provide useful and accurate predictions for further observations.

One of the main problems, or perhaps "the" main problem, of numerical cosmology is to provide simulations with a simple but accurate feedback scheme able to give results in agreement in observations. This has proven to be an outstanding problem, since it is practically impossible to create recipes for feedback that take into account all the physical processes involved in the game, ranging from star formation in molecular clouds, to the escape of matter and radiation from galaxies.

We created a new feedback scheme to be applied to high resolution numerical simulations of galaxy formation in a cosmological context. Our prescriptions are straightforward enough to be applied to a set of large simulations, but at the same time contain all the relevant physics necessary to describe the transport of matter from the galaxies to the diffuse intergalactic medium. The use of semi-analytic recipes for the physics of baryons applied to pure N-body simulations allows us to find a good compromise between high mass resolution and a large simulated volume: while a high resolution in mass is crucial to determine the role of galaxies with different masses in polluting the IGM with the 
products of stellar nucleosynthesis, a large region is necessary to study the effects of winds in their proper cosmological context.

In section 4 we present results for the volume filling factor of galactic winds, which gives us an estimate of the probability of finding disturbances in the Ly $\alpha$ forest due to winds (Bertone, Stoehr \& White 2005). We then extract synthetic spectra from our simulated region and directly investigate the effects of feedback on the statistical properties of the Ly $\alpha$ forest, and in particular on the flux probability distribution function (PDF).

We suggest an explanation for the Adelberger effect in section 5. Adelberger et al. (2003) find that the transmitted flux in the surroundings of high redshift galaxies is strongly enhanced and suggest that this may be caused by the presence of winds outflowing from the galaxies. However, current simulations seem to be unable to reproduce the effect.

\section{The simulations}

Our "M3" set of N-body simulations (Stoehr 2003) is a re-simulation at higher mass resolution of an approximately spherical region of space with diameter $52 h^{-1} \mathrm{Mpc}$. We assume a $\Lambda$ CDM cosmology with matter density $\Omega_{m}=0.3$, dark energy density $\Omega_{\Lambda}=0.7$, Hubble constant $h=0.7$, primordial spectral index $n=1$, and normalisation $\sigma_{8}=0.9$. The high resolution region contains about 70 million dark matter particles of mass $1.7 \times 10^{8} \mathrm{M}_{\odot}$. This region, which has a mean density close to the cosmic mean and contains a majority of field galaxies, is well suited for investigations of the IGM. The formation and evolution of galaxies is given by the semi-analytic model of Springel et al. (2001). We add our recipes for winds on top of this pre-existing scheme.

We model galactic winds as uniform pressure-driven bubbles of hot gas emerging from star forming galaxies. The adiabatic phase is terminated when the loss of energy by radiation becomes substantial, that is, most of the energy transferred to the swept-up gas is radiated away. When the cooling time of the hot bubble becomes shorter than the age of the wind, a thin shell of cooled gas forms near the bubble's outer boundary and continues to expand, pushed by the momentum input from the wind. We do not resolve the details of the first phases of wind evolution, when the superbubbles blow out of the ISM of galaxies. Instead, we want to investigate the effects of the long-term evolution of winds on the IGM once they have escaped the visible regions of galaxies. We make the simplifying assumption of spherical symmetry for the wind evolution. This may seem a rough approximation at $z \sim 0$, where outflows are mostly bipolar, but it is a good assumption at higher redshifts, where outflows seem to have a more spherical geometry (Shapley et al. 2003). The adiabatic, pressure-driven phase of the wind evolution is described by the equation for the conservation of energy (Ostriker \& McKee 1988):

$$
\frac{d E}{d t}=\frac{1}{2} \dot{M}_{w} v_{w}^{2}+\varepsilon 4 \pi R^{2} \cdot\left\{\left[\frac{1}{2} \rho_{o} v_{o}^{2}+u_{o}-\rho_{o} \frac{G M_{h}}{R}\right]\left(v_{s}-v_{o}\right)-v_{o} P_{o}\right\},
$$

while the momentum-driven phase is described by the equation for the conservation of momentum:

$$
\frac{d}{d t}\left(m v_{s}\right)=\dot{M}_{w}\left(v_{w}-v_{s}\right)-\frac{G M_{h}}{R^{2}} m-\varepsilon 4 \pi R^{2}\left[P_{o}+\rho_{o} v_{o}\left(v_{s}-v_{o}\right)\right] .
$$

$R$ and $v_{s}$ are the radius and the velocity of the shock, $\dot{M}_{w}$ and $v_{w}$ the mass outflow rate and the outflow velocity of the wind, $\rho_{o}, P_{o}$, and $v_{o}$ the density, the pressure, and the outward velocity of the surrounding medium, and $M_{h}$ the total mass internal to the shock radius. The parameter $m$ is the mass of the shell. $\varepsilon$ is a parameter, the entrainment 
fraction, that defines the fraction of mass that the wind sweeps up while crossing the ambient medium. A more detailed description of our prescriptions for galactic winds can be found in Bertone et al. (2005).

\section{Wind dynamics}

In our simulations both bursts of star formation and quiescent star formation may be able to power the winds, since we do not put constraints on the star formation rate to allow galaxies to blow winds. It is not possible to predict a priori when a wind will escape the gravitational pull of a galaxy, since its evolution and its final fate are linked to several factors, like the star formation and the mass accretion history of the galaxy, the potential well of the dark matter halo in which it expands, the amount of mass accreted from the wind and from the IGM, and so on.

In general, a wind receives energy from the starburst and is slowed down by the gravitational attraction of the central galaxy and by the ram pressure of the ambient medium. Thermal pressure effects are considerable inside cluster halos, but negligible in the IGM. If the entrained mass is small (e.g. $\varepsilon=0.1$ ), a large fraction of the bubble or shell mass is composed by the supernova ejecta and shocked ISM in the wind fluid, which are outflowing from the galaxy with a velocity often much larger than the escape velocity of the halo. Since little energy or momentum has to be spent by the wind to accelerate the entrained mass, the shock velocity is less sensitive to energy losses by pressure and gravity. Such a wind has thus a higher probability to overcome the gravitational pull and break free from the halo than more mass loaded winds. When the mass loading is substantial (e.g. $\varepsilon=0.3$ ), a significant part of the wind energy is consumed to accelerate the entrained gas and the expansion slows down considerably. If the amount of energy spent to accelerate the swept-up mass is large compared to the total energy in the wind, the shock velocity may become lower than the escape velocity of the galaxy and the wind collapses back onto the galaxy.

At high redshift winds tend to be mostly momentum-driven, while at lower redshifts bubbles have a much higher probability of remaining adiabatic. This is partly due to the higher mean density of the Universe at high $z$ and partly to a lower energy input from star formation, which determines lower bubble temperatures and shorter cooling times immediately after blowout. The transition from pressure-driven bubbles to momentumdriven shells may be a first hint that a wind is not powerful enough to escape the galaxy attraction. In fact, pressure-driven winds are overall more likely to escape galaxies than momentum-driven ones.

\section{Volume filling factor of galactic winds}

In Fig. 1 we show an example of the evolution of winds from $z=5$ to $z=1$. A thin slice is cut through the central plane of the simulation and the density distribution of the gas in the slice is shown.

An estimate of the volume filling factor $f_{v}$ of galactic winds at the redshifts where absorption in quasar spectra is observed can be translated into an estimate of the probability to find disturbances in the Ly $\alpha$ forest due to feedback effects and, in particular, to the presence of wind bubbles and shells. Disturbances here mean regions of the spectra where there are significant variations in the optical depth, due to non-gravitational processes stirring the IGM. We calculate the filling factor of winds as the ratio between the volume affected by winds and the total volume of our simulated region. In Fig. 2 we show the behaviour of $f_{v}$ as a function of time and model parameters. The smaller values 


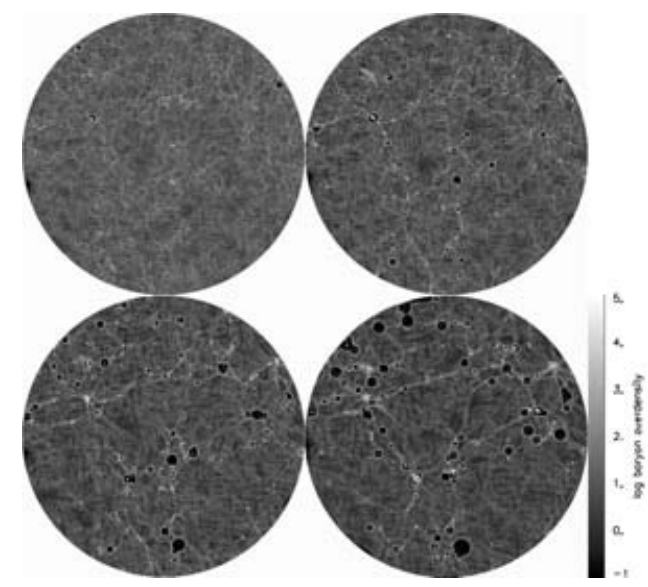

Figure 1. Structure of wind-filled regions as a function of time. M3 simulations with parameters $\varepsilon=0.3$ and $K=0.5$. From top to bottom and left to right, the redshifts of the snapshots are respectively $z=5, z=3$, $z=2$, and $z=1$. The diameter of the region shown is $52 h^{-1}$ co-moving Mpc. The white contours show the extension of winds, while the dark regions inside the contours are the regions depleted of gas by outflows.

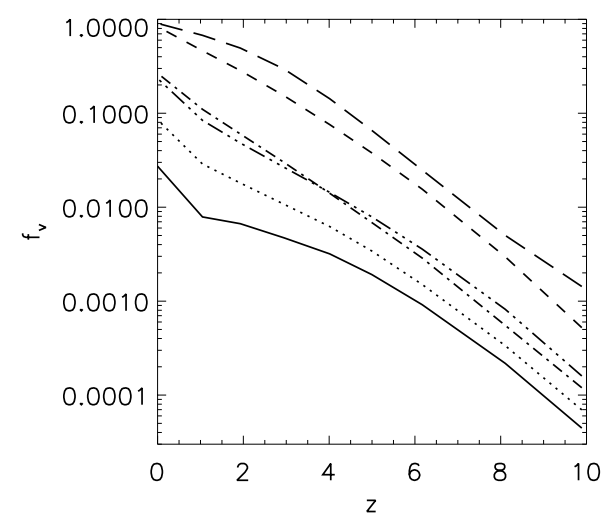

Figure 2. Volume filling factor of winds in M3 as a function of redshift and model parameters. The lines represent different combinations of our model parameters: (1) solid line: $K=0.5, \varepsilon=1$; (2) dotted line: $K=0.5$, $\varepsilon=0.6$; (3) dashed line: $K=0.1, \varepsilon=0.3$; (4) dashed dotted line: $K=0.5, \varepsilon=0.3 ;(5)$ dashed three-dotted line: $K=1, \varepsilon=0.3$; (6) long dashed line: $K=0.5, \varepsilon=0.1$.

for $f_{v}$ are clearly related to the cases where fewer mass loaded bubbles are formed and expand into the IGM and vice-versa.

\section{Synthetic Ly $\alpha$ spectra}

While our estimate of the volume filling factor is a theoretical prediction of the probability to find disturbances in the Ly $\alpha$ forest due to feedback effects, we can test our predictions by extracting a set of artificial spectra from our simulated region and analysing its statistical properties.

We first extract a set of about 100 spectra along random directions through our simulated region. The length of each spectrum varies according to the position of the line of sight (LoS) across the sphere and ranges from about 60 to $150 \AA$ A. In Fig. 3 we show the PDF of the flux in our spectra and we compare it to the observational results of McDonald et al. (2000). The striking result from Fig. 3 is the almost perfect match between the PDF of the set of spectra perturbed by winds and the PDF of a second set of unperturbed spectra. The two sets were extracted along the same random directions, and are therefore directly comparable to each other. This demonstrates that feedback effects from galactic winds do not significantly modify the statistical properties of the Ly $\alpha$ forest and confirms the predictions we have made on the basis of our results for the volume filling factor. The PDF of the simulated spectra differs slightly from the observational results at high fluxes, while the agreement is accurate everywhere else. The difference at $F \sim 0.9$ is most likely due to the inability of our semi-analytic prescriptions to capture the distribution of the hot and cold gas in galaxies and halos. This particularly affects the absorption lines associated with high density systems.

Although the statistical properties of the forest are globally unchanged by winds, individual signatures of bubbles and shells can be clearly recognised when perturbed and 


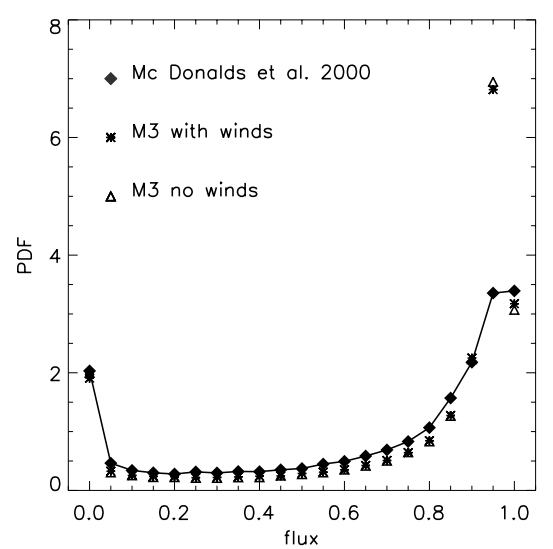

Figure 3. The flux probability distribution function for our set of simulated spectra, compared to the observations of McDonald et al. (2000). The presence of winds in our simulated box does not produce any significant variation in the statistical properties of the Ly $\alpha$ forest.

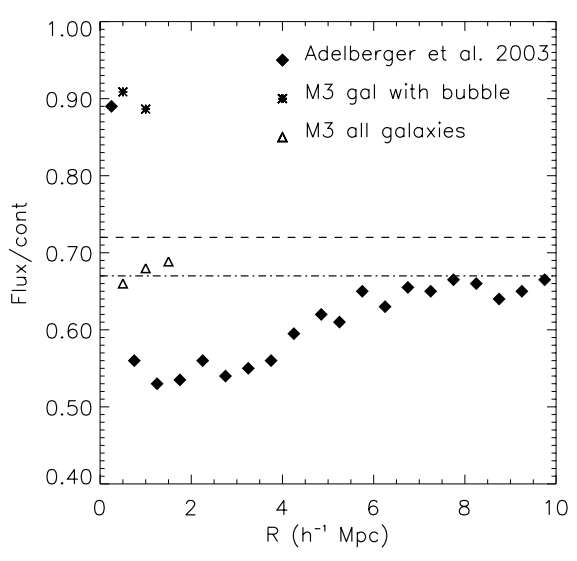

Figure 4. The increase of the mean transmitted flux around LBGs, as observed by Adelberger et al. (2003), may be explained by the presence of hot wind bubbles around the galaxies in the sample. Momentum-driven winds alone are unable to explain the effect, which may indicate that the results of Adelberger and collaborators are the result of a selection effect.

unperturbed spectra are compared one by one. In Fig. 5 we show an example of a LoS that crosses a pressure-driven bubble at increasing distances from the galaxy centre. The flux transmissivity inside the bubble is strongly increased by the presence of the hot ionised bubble. In Fig. 6 we show instead the effect of a momentum-driven shell. The cooled wind cavity does produce an increase in the flux transmissivity, but the effect is not as striking as for pressure-driven bubbles. More significantly, the cold and dense material accumulated in the shell produces narrow and saturated absorption lines 1 to 3 $\AA$ wide.

Since it is common in our spectra to observe an increased flux transmissivity around bright star forming galaxies, we conclude that the presence of hot wind bubbles around star forming galaxies may be a possible explanation for the observational results of Adelberger et al. (2003), who find an almost complete transmission of the Ly $\alpha$ continuum in the proximity of high redshift Lyman break galaxies. In Fig. 4 we show our results for pressure-driven bubbles and for normal bright galaxies compared to the data of Adelberger et al. (2003).

\section{Conclusions}

Our estimate of the volume filling factor of winds and the PDF of the flux in a sample of artificial spectra extracted from our simulated region confirm the idea that galactic outflows do not significantly modify the statistical properties of the Ly $\alpha$ forest.

However, galactic winds do leave individual detectable signatures in the Ly $\alpha$ forest, in the form of an increased flux transmissivity inside pressure-driven bubbles and narrow, saturated absorption lines due to cooled, momentum-driven shells. We argue that the Adelberger effect can be explained by the presence of hot bubbles around the observed LBGs. However, in our simulations only about $20 \%$ of winds are pressure-driven at $z \sim 3$ and the observations of Adelberger may therefore be the result of a biased selection of 


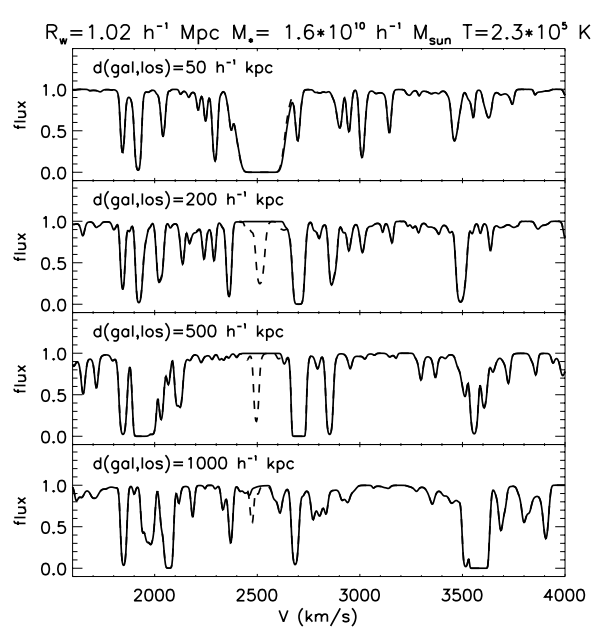

Figure 5. The effect of a pressure-driven bubble along the LoS to a quasar: the Ly $\alpha$ flux is almost completely transmitted inside the hot ionised bubble, as predicted by the observations of Adelberger et al. (2003). Solid line: perturbed spectrum; dashed line: unperturbed spectrum.

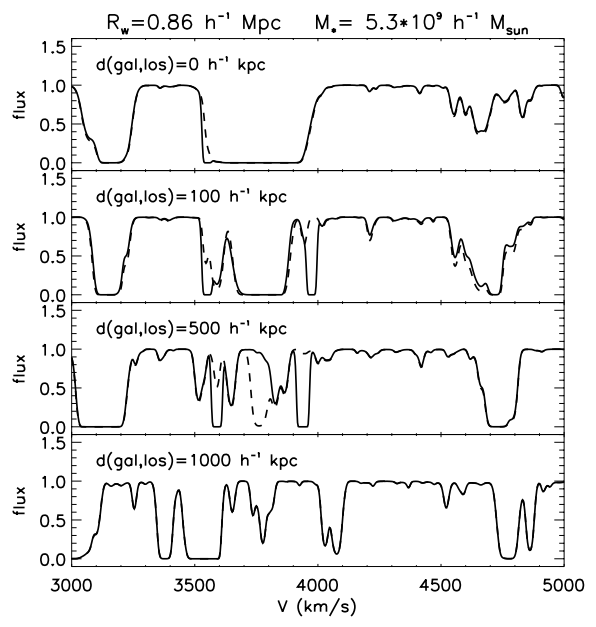

Figure 6. When a LoS crosses a momentum-driven wind, two narrow and saturated absorption lines are produced, while the flux in the cooled wind cavity may produce a slight increase in the transmitted flux. Solid line: perturbed spectrum; dashed line: unperturbed spectrum.

the target galaxies: indeed, the three galaxies in the sample can hardly be representative of the full population. The fact that we can explain the Adelberger effect as caused by pressure-driven winds, while so far simulations have been unable to reproduce the result by using a momentum-driven approximation for winds, may be a clear indication that a more sophisticated and physically motivated model for the physics of galactic winds is necessary, if we want to be able to reproduce the full range of observational results with any accuracy.

\section{Acknowledgements}

We thank P. A. Thomas for helpful discussions and J.-S. Huang for support and encouragement. This work has been supported by the Research and Training Network "The Physics of the Intergalactic Medium" set up by the European Community under contract HPRN-CT-2000-00126. S. B. was partially supported by PPARC.

\section{References}

Adelberger, K. L., Steidel, C. C., Shapley, A. E., Pettini, M., 2003, ApJ, 584, 45

Bertone, S., Stoehr, F., White S. D. M., 2005, MNRAS, in press

McDonald, P., Miralda-Escudé, J., Rauch, M., Sargent, W. L. W., Barlow, T. A., Cen, R., Ostriker, J. P., 2000, ApJ, 543, 1

Ostriker, J. P., McKee, C. F., 1988, Rev. Mod. Phys., 60, 1

Shapley, A. E., Steidel, C. C., Pettini, M., Adelberger, K. L., 2003, ApJ, 588, 65

Springel, V., White, S. D. M., Tormen, G., Kauffmann, G., 2001, MNRAS, 328, 726

Stoehr, F., 2003, PhD Thesis, Ludwig Maximilian Universität, München 